\title{
ASYMPTOTIC EXPANSIONS OF SOME INTEGRAL TRANSFORMS BY USING GENERALIZED FUNCTIONS \\ BY
}

\author{
AHMED I. ZAYED
}

\begin{abstract}
ABSTRACr. The technique devised by Wong to derive the asymptotic expansions of multiple Fourier transforms by using the theory of Schwartz distributions is extended to a large class of integral transforms. The extension requires establishing a general procedure to extend these integral transforms to generalized functions. Wong's technique is then applied to some of these integral transforms to obtain their asymptotic expansions. This class of integral transforms encompasses, among others, the Laplace, the Airy, the $K$ and the Hankel transforms.
\end{abstract}

1. Introduction. Recently, the theory of generalized functions has been introduced into the field of asymptotic expansions of integral transforms; see $[18,19,6]$.

One of the advantages of this approach is to be able to interpret and to assign values to some divergent integrals.

This approach goes back to Lighthill [10] who was the first to use generalized functions to study the asymptotic expansions of Fourier transforms.

Borrowing Lighthill's idea, several people were able to derive asymptotic expansions for other types of integral transforms such as Laplace [2], Stieltjes [11], Riemann-Liouville fractional integral transforms [12] and Mellin convolution of two algebraically dominated functions [17].

The use of generalized functions in the theory of asymptotic expansions has another advantage; that is, in some cases it can provide explicit expressions for the error terms in shorter and more direct ways than the classical methods $[9,11]$. However, one should not overemphasize the importance of the generalized functions approach since, for the most part, it is an alternative but not an exclusive technique.

The use of generalized functions, except for the case of Fourier transform, was basically limited to integral transforms with exponentially decaying kernels.

In a recent paper [18], Wong devised a new technique based on the theory of Schwartz distributions to provide an alternative derivation to the one given in [15] for the asymptotic expansions of multiple Fourier transforms. In doing that, he actually showed that the generalized functions method could be applied to integral transforms with oscillatory kernels with equal advantage to the classical methods.

The analysis of his technique shows that the theory of Schwartz distributions and their Fourier transforms [14] plays a vital role in the proof. Therefore, it seems inevitable that extending Wong's technique to other types of integral transforms

Received by the editors March 9, 1981 and, in revised form, July 20, 1981.

1980 Mathematics Subject Classification. Primary 41A60; Secondary 46F12.

Key words and phrases. Asymptotic expansion, generalized function, integral transform.

(C)1982 American Mathematical Society 0002-9947/81/0000-0657/\$06.00 
requires a similar theory that would extend these different types of integral transforms to generalized functions in a unified way. As far as I am aware, except of some earlier results obtained by Jones [22], such a theory has not been developed yet.

Therefore, the purpose of this paper is twofold:

(i) To establish a general procedure to extend certain integral transforms to generalized functions.

(ii) To apply Wong's technique to these transforms in order to derive their asymptotic expansions.

The class of integral transforms we shall consider contains, among others, Laplace, Mellin, Hankel, Stieltjes, Weierstrass, Airy and the $K_{\mu}$ transforms.

In fact, the procedure may be applied to any integral transform of the type,

$$
\mathscr{F}(f)(s)=F(s)=\int_{I} f(t) K(s, t) d t
$$

where the kernel $K(s, t)$ is an infinitely differentiable function that can be imbedded in some testing-function space and $I=(a, b)$ where $-\infty \leqslant a<b \leqslant \infty$.

However, when we study the asymptotic expansions of some of these transforms, we shall restrict our investigation to the case where $I=(0, \infty)$ and to the behavior of $\mathscr{F}(f)(s)$ as $s \rightarrow \infty$.

2. Preliminaries. In this section, we introduce some of the notation and the terminology that will be used.

Throughout this paper, $I$ will denote the open interval $(a, b)$ where $-\infty \leqslant a<b$ $\leqslant \infty$ and $C^{\infty}(I)$ will denote the set of all infinitely differentiable functions on $I$.

A conventional function (ordinary function) on $I$ means a function whose domain is $I$ and whose range is a subset of the real line or the complex plane. A generalized function means a functional on some testing-function space. In general, if a certain concept has been extended from conventional functions to generalized functions, we shall use the adjectives "conventional" and "generalized" to distinguish between the two since they are not always the same, e.g., conventional (generalized) derivatives and conventional (generalized) integral transforms,... etc.

A locally integrable function on $I$ means a conventional function that is Lebesgue integrable on every open subinterval $J$ of $I$ whose closure $\bar{J}$ is contained in $I$.

A countably normed space $V$ is a topological vector space whose topology is generated by a countable family of separating seminorms. A countable-union space $V$ is a countable union of countably normed spaces $\left\{V_{n}\right\}_{n=1}^{\infty}$ such that

$$
V=\bigcup_{n=1}^{\infty} V_{n}, \quad V_{n} \subset V_{n+1} \text { for } n=1,2,3, \ldots,
$$

and the topology of $V_{n}$ is stronger than the topology it inherits from $V_{n+1}$. The topology of $V$ is the inductive limit topology $[3,8]$. Let $V$ be either a countably normed space or a countable-union space. Let all the members of $V$ be in $C^{\infty}(I)$. Consider the sequence $\left\{\phi_{n}(t)\right\}_{n=1}^{\infty}$ in $V$. If the convergence of the sequence $\left\{\phi_{n}(t)\right\}_{n=1}^{\infty}$ in $V$ implies the convergence of the sequences $\left\{d^{k} \phi_{n}(t) / d t^{k}\right\}_{n=1}^{\infty} ; k=0,1,2, \ldots$, on 
every compact subset of $I$, then we say that $V$ is a testing-function space.

Members of the dual space $V^{*}$ of a testing-function space $V$ are called generalized functions. A conventional function $f(t)$ on $I$ that satisfies the following two conditions is called a regular member of $V^{*}$ :

(i) $\int_{I} f(t) \phi(t) d t$ exists in the Lebesgue sense for every $\phi \in V$.

(ii) If $\phi_{n}(t) \rightarrow 0$ in $V$ as $n \rightarrow \infty$, then $\int_{I} f(t) \phi_{n}(t) d t \rightarrow 0$ as $n \rightarrow \infty$.

3. Spaces of generalized functions. The procedure we shall establish here is valid for any interval $I=(a, b)$; however, in most cases of interest, $I$ will be either of the form $(0, \infty)$ or $(-\infty, \infty)$.

Let $K(s, t)$ be the kernel of the integral transform of equation (1.1). We assume that $K(s, t)$ is a $C^{\infty}$-function for $(s, t) \in J \times I$ where $J$ is an open subinterval of $I$. If $s$ is complex, we require that $\operatorname{Re} s$ be in $J$.

The space $\mathbb{Q}$ of $\mathscr{F}$-transformable functions is defined by $\mathbb{Q}=\{f(t) \mid$ the integral $\int_{I} f(t) K(s, t) d t$ exists and is finite for sufficiently large $\left.\operatorname{Re} s\right\}$.

The existence of the integral is understood to be in the following sense:

(i) If $I=(0, \infty)$, then $\lim _{c \rightarrow \infty} \int_{0}^{c} f(t) K(s, t) d t<\infty$.

(ii) If $I=(-\infty, \infty)$, then $\lim _{c \rightarrow \infty} \int_{-c}^{c} f(t) K(s, t) d t<\infty$.

Let $\eta(t, a, b)$ be a continuous function on $I$ for some real parameters $a$ and $b$ such that $\eta(t, a, b) \neq 0$ for any $t \in I$.

Let $R$ denote a linear differential operator of the form

$$
R=\theta_{0} D^{n_{1}} \theta_{1} D^{n_{2}} \theta_{2} \cdots D^{n_{p}} \theta_{p}
$$

where $D=d / d t$, the $n_{k}$ 's are positive integers and the $\theta_{k}(t)$ 's are $C^{\infty}$-functions on $I$ that are, together with their derivatives of all orders, never equal to zero therein. We define the space $\mathcal{L}_{a, b}$ as follows:

The space $\mathcal{L}_{a, b}$ consists of all complex-valued $C^{\infty}$-functions $\phi(t)$ on $I$ on which the functionals $\gamma_{k, a, b}$ defined by

$$
\gamma_{k, a, b}(\phi)=\sup _{t \in I}\left|\eta(t, a, b) R^{k} \phi(t)\right|, \quad k=0,1,2 \ldots,
$$

assume finite values. For fixed $a$ and $b$, we write $\gamma_{k}(\phi)$ instead of $\gamma_{k, a, b}(\phi)$. If $\eta$ depends only on one parameter (say $a$ ), we write $\varrho_{a}$ for the underlying space. Clearly, $\varrho_{a, b}$ is a linear space under the pointwise addition of functions and multiplication by complex numbers. The collection $\left\{\gamma_{k}\right\}_{k=0}^{\infty}$ is a separating family of seminorms since each $\gamma_{k}(k=1,2, \ldots)$ is a seminorm and $\gamma_{0}$ is a norm. We adopt the standard notation that $\mathscr{E}(I)$ is the space of $C^{\infty}$-functions on $I$ provided with the topology induced by the seminorms.

$$
\gamma_{k, K}(\phi)=\sup _{t \in K}\left|D^{k} \phi(t)\right|, \quad \phi(t) \in C^{\infty}(I),
$$

where $k$ is a nonnegative integer and $K$ is a compact subset of $I$, while $\mathscr{D}(I)$ is the space of all $C^{\infty}$-functions $\phi(t)$ on $I$ that vanish outside some compact subset of $I$ (not necessarily the same subset for all of them). The topology of $\mathscr{D}(I)$ is described as follows: the sequence $\left\{\phi_{n}\right\}_{n=1}^{\infty}$ converges to zero in $\mathscr{D}(I)$ if and only if all the $\phi_{n}$ 's vanish outside some compact subset $K$ of $I$ and converge uniformly to zero together with all their derivatives on $K$. 
The dual spaces of the spaces $\mathscr{D}(I), \mathfrak{L}_{a, b}$ and $\mathcal{E}(I)$ will be denoted by $\mathscr{D} *(I), \mathfrak{L}_{a, b}^{*}$ and $\mathcal{E}^{*}(I)$ respectively. Some properties of the space $\mathcal{L}_{a, b}$ and its relationship with the spaces $\mathscr{D}(I)$ and $\mathscr{E}(I)$ are given in the following lemma.

LEMMA 3.1. (i) $\mathfrak{L}_{a, b}$ is complete and therefore a Fréchet space.

(ii) $\mathscr{D}(I) \subset \mathfrak{L}_{a, b} \subset \mathcal{E}(I)$. In fact, $\mathfrak{Q}_{a, b}$ is dense in $\mathcal{E}(I)$.

(iii) If $f(t)$ is a locally integrable function on I such that $f(t) / \eta(t, a, b)$ is absolutely integrable on $I$, then $f(t) \in \mathfrak{L}_{a, b}^{*}$ and $\langle f, \phi\rangle=\int_{I} f(t) \phi(t) d t$ for any $\phi \in \mathfrak{L}_{a, b}$.

Proof. Let $\left\{\phi_{\nu}\right\}_{\nu=1}^{\infty}$ be a Cauchy sequence in $\mathcal{L}_{a, b}$ and let $K$ be an arbitrary compact subset of $I$. In view of the seminorm $\gamma_{1}, R^{1} \phi_{\nu}(t)$ converges uniformly on $K$. Let $T$ be a fixed point in $I$ and let $D^{-1}$ denote the integration operator

$$
D^{-1}=\int_{T}^{t} \cdots d x
$$

Thus, for any differentiable function $g(t)$ on $I$, we have $D^{-1} D g(t)=g(t)-g(T)$. Since uniform convergence on compact sets is preserved under multiplication by $C^{\infty}$-functions and under the application of $D^{-1}$, it follows from the definition of $R$ that

$$
D^{-n_{1}} \theta_{0}^{-1} R \phi_{\nu}(x)=\left(\theta_{1} D^{n_{2}} \cdots D^{n_{p}} \theta_{p}\right) \phi_{\nu}(x)+P_{\nu}(x)
$$

converges uniformly on $K$, where $P_{\nu}(x)$ is a polynomial of degree less than $n_{1}$ with $P_{\nu}(T)=0$.

Repeating this argument yields

$$
R^{k} \phi_{\nu}(x)=R^{-1} R^{k+1} \phi_{\nu}(x)+\sum_{j<\sigma} a_{\nu j} g_{j}(x)
$$

where $\sigma=n_{1}+n_{2}+\cdots+n_{p}, a_{\nu j}$ are constants, $g_{j}(x)$ are antiderivatives of $C^{\infty}$ functions and

$$
R^{-1}=\theta_{p}^{-1} D^{-n_{p}} \cdots D^{-n_{1}} \theta_{0}^{-1} .
$$

On observing that $R^{-1} R^{k+1} \phi_{\nu}(x)$ vanishes at $x=T$ together with all its derivatives of order less than $\sigma$, one can easily see that the sum in equation (3.4) is that solution of the differential equation $R y=0$ whose derivatives at $T$ of order less that $\sigma$ are the same as those of $R^{k} \phi_{\nu}(x)$. Since $R^{k} \phi_{\nu}(x)$ and $R^{-1} R^{k+1} \phi_{\nu}(x)$ converge uniformly on compact sets, then so does $\Sigma_{j<\sigma} a_{v j} g_{j}(x)$. The $g_{j}(x)$ can be chosen so that they are linearly independent and hence $\lim _{\nu \rightarrow \infty} a_{v j}$ exists and is equal to say $a_{j}$ [5]. Setting $k=0$ and taking the derivative of equation (3:4), we obtain

$$
\begin{aligned}
D \phi_{\nu}(x)= & {\left[\left(D \theta_{p}^{-1}\right) D^{-n_{p}}+\theta_{p}^{-1} D^{1-n_{p}}\right] \theta_{p-1}^{-1} D^{-n_{p-1}} \cdots D^{-n_{1}} \theta_{0}^{-1} R \phi_{\nu}(x) } \\
& +\sum_{j<\sigma} a_{\nu j} D g_{j}(x) .
\end{aligned}
$$

Since the right-hand side of equation (3.6) converges uniformly on $K$, it follows that $D \phi_{\nu}(x)$ also converges uniformly on $K$. By repeating this procedure, one can show the uniform convergence on $K$ of the sequences $\left\{D^{j} \boldsymbol{\phi}_{\nu}(x)\right\}_{\nu=1}^{\infty}, j=2,3, \ldots, \sigma$. Once more, if we set $k=1$ in (3.4) (the $a_{v j}$ are in general different constants) and 
repeat the previous argument, we can easily show that $\left\{D^{j} R \phi_{\nu}(x)\right\}_{\nu=1}^{\infty}$ converges uniformly on $K$ for $j=1,2, \ldots, \sigma$. From this, we obtain successively the uniform convergence on $K$ of the sequences $\left\{D^{j} \phi_{\nu}(x)\right\}_{\nu=1}^{\infty}$ for $j=\sigma+1, \sigma+2, \ldots, 2 \sigma$.

Continuing in this fashion, we can show the uniform convergence on $K$ of the sequences $\left\{D^{j} \phi_{\nu}(x)\right\}_{\nu=1}^{\infty}, j=0,1,2, \ldots$, and hence the existence of a $C^{\infty}$-function $\phi(x)$ on $I$ such that

$$
\lim _{\nu \rightarrow \infty} D^{j} \phi_{\nu}(x)=D^{j} \phi(x)
$$

uniformly on any compact subset of $I$ for $j=0,1,2, \ldots$ In fact, one can easily show now that

$$
\lim _{\nu \rightarrow \infty} R^{k} \phi_{\nu}(x)=R^{k} \phi(x)
$$

uniformly on compact subsets of $I$ for any nonnegative integer $k$.

Since $\left\{\phi_{\nu}\right\}_{\nu=1}^{\infty}$ is a Cauchy sequence in $\varrho_{a, b}$, then for any $\varepsilon>0$, there exists an $N(k)$ such that for every $\nu, \mu>N(k)$

$$
\gamma_{k, a, b}\left(\phi_{\nu}-\phi_{\mu}\right)<\varepsilon
$$

Therefore, when $\mu \rightarrow \infty$, we have $\gamma_{k, a, b}\left(\phi_{\nu}-\phi\right)<\varepsilon$ for all $\nu>N(k)$. That is $\gamma_{k, a, b}\left(\phi_{\nu}-\phi\right) \rightarrow 0$ as $\nu \rightarrow \infty$ for every $k$. Finally, there exists a constant $C_{k}$ independent of $\nu$ such that $\gamma_{k, a, b}\left(\phi_{\nu}\right)<C_{k}$.

Therefore, by equation (3.9), we have

$$
\gamma_{k, a, b}(\phi) \leqslant \gamma_{k, a, b}\left(\phi_{\nu}\right)+\gamma_{k, a, b}\left(\phi-\phi_{\nu}\right) \leqslant C_{k}+\varepsilon
$$

which implies that $\phi \in \mathcal{L}_{a, b}$ and that finishes the proof.

(ii) That $\mathscr{D}(I)$ is a subspace of $\mathcal{L}_{a, b}$ is trivial and left to the reader. By definition, $\mathcal{L}_{a, b}$ is a subset of $\mathcal{E}(I)$. Thus, what remains to be proved is that the topology of $\mathcal{E}_{a, b}$ is stronger than the topology it inherits from $\mathcal{E}(I)$. But this was shown in the proof of part (i) (see equation (3.7)). That $\mathcal{L}_{a, b}$ is dense in $\mathcal{E}(I)$ follows from the fact that $\mathcal{D}(I)$ is dense in $\mathcal{E}(I)$.

(iii) Indeed,

$$
|\langle f, \phi\rangle|=\left|\int_{I} \frac{f(t)}{\eta(t, a, b)} \eta(t, a, b) \phi(t) d t\right| \leqslant \gamma_{0, a, b}(\phi) \int_{I}\left|\frac{f(t)}{\eta(t, a, b)}\right| d t<\infty .
$$

Q.E.D. [21].

3.1. In general, $\mathscr{D}(I)$ is not dense in $\mathcal{L}_{a, b}$ and counterexamples can be found in

3.2. Let us assume that for $a \leqslant c$ and $d \leqslant b$, we have $0<\eta(t, a, b) \leqslant \eta(t, c, d)$ on I. Then it follows that $\mathcal{L}_{c, d} \subset \mathcal{E}_{a, b}$ and that convergence in $\mathcal{L}_{c, d}$ implies convergence in $\varrho_{a, b}$. This can be seen from the relation

$$
\gamma_{k, a, b}(\phi)=\sup _{t \in I}\left|\eta(t, a, b) R^{k} \phi(t)\right| \leqslant \sup _{t \in I}\left|\eta(t, c, d) R^{k} \phi(t)\right|=\gamma_{k, c, d}(\phi) .
$$

3.3. Let $w$ and $z$ be such that $-\infty \leqslant w<z \leqslant \infty$. Choose two monotonic sequences of real numbers $\left\{a_{\nu}\right\}_{\nu=1}^{\infty}$ and $\left\{b_{\nu}\right\}_{\nu=1}^{\infty}$ such that $a_{\nu} \rightarrow w^{+}$and $b_{\nu} \rightarrow z^{-}$. Under the same assumption of the preceding remark, we can define a countable-union space $\mathcal{L}(w, z)$ $=\cup_{\nu=1}^{\infty} \mathcal{L}_{a_{\nu}, b_{\nu}}$. 
One can easily verify the following:

(i) The definition of $\mathcal{E}(w, z)$ is independent of the choices of $\left\{a_{\nu}\right\}_{\nu=1}^{\infty},\left\{b_{\nu}\right\}_{\nu=1}^{\infty}$ and if $w \leqslant u, v \leqslant z$, then $\mathcal{L}(u, v) \subset \mathcal{L}(w, z)$. Moreover, $\mathcal{L}(w, z) \subseteq \mathcal{L}_{w, z}$.

(ii) A sequence $\left\{\phi_{\nu}\right\}_{\nu=1}^{\infty}$ converges in $\mathcal{L}(w, z)$ if and only if it converges in one of the spaces $\mathcal{L}_{a_{v}, b_{v}}$. In addition to that, $\mathcal{L}(w, z)$ is complete and so is its dual space $\mathcal{L}^{*}(w, z)[\mathbf{2 1}]$.

4. Continuous operators on the dual space. Let $X$ stand for either $\mathcal{L}_{a, b}$ or $\mathcal{L}(a, b)$. Accordingly, let $Y$ be a countably normed space or a countable-union space. Also, let $Q$ be a continuous linear operator from $X$ into $Y$. Define the adjoint operator $Q^{*}$ of $\mathcal{Q}$ on the dual space $Y^{*}$ of $Y$ by

$$
\left\langle\mathbb{Q}^{*} f, \phi\right\rangle=\langle f, \mathbb{Q} \phi\rangle
$$

where $f \in Y^{*}$ and $\phi$ traverses all of $X$.

It is well known that $Q^{*}$ is a continuous linear operator from $Y^{*}$ into $X^{*}$. In most cases of interest, $Y^{*}$ will be a space of generalized functions.

We should emphasize the fact that unlike the spaces $\mathscr{D}(I)$ and $\mathscr{E}(I)$, the space $X$ is not necessarily closed under multiplication by $C^{\infty}$-functions. Moreover, differentiation may not be a continuous operator on $X$. However, $R$ is certainly a continuous linear operator from $X$ into itself. For

$$
\gamma_{k}(R \phi)=\sup _{t \in I}\left|\eta(t) R^{k} R \phi(t)\right|=\gamma_{k+1}(\phi)
$$

4.1. If $f(t)$ is a regular member of $X^{*}$, then the adjoint operator $R^{*}$ of $R$ takes the form

$$
\left\langle R^{*} f, \phi\right\rangle=\langle f, R \phi\rangle=\int_{I} f(t) R \phi(t) d t .
$$

In addition, if we assume that $f \in C^{\sigma}(I)$ and either $f^{(j)}(a)=0=f^{(j)}(b)$ for $j=$ $0,1,2, \ldots, \sigma-1$ or $\phi^{(k)}(a)=0=\phi^{(k)}(b)$ for $k=0,1,2, \ldots$ and all $\phi$ in $X$, then successive integration by parts gives

$$
\left\langle R^{*} f, \phi\right\rangle=\int_{I} \phi(t)\left[\theta_{p} D^{n_{p}} \cdots D^{n_{1}} \theta_{0}\right] f(t) d t
$$

where $\sigma=n_{1}+n_{2}+\cdots+n_{p}$.

This suggests defining $R^{*}$ as the operator

$$
R^{*}=\theta_{p} D^{n_{p}} \cdots D^{n_{1}} \theta_{0}
$$

where $D$ denotes the conventional differentiation operator. This definition will also be used even when $f(t)$ is an arbitrary member of $X^{*}$, but in this case, $D$ will denote the generalized derivative. Needless to say, the conventional and the generalized derivatives need not be equal.

If $R_{c}$ and $R_{g}$ denote the conventional and the generalized operator $R$ respectively, then in general $R_{c} \neq R_{g}$. But under the same assumptions of remark (4.1), the relation $R_{c}=R_{g}$ is valid. 
5. Generalized integral transforms. The extension of the classical integral transforms to generalized functions has been developed by many people in the last three decades. See [21] for a bibliography. Unfortunately, there is no universal agreement on how to extend a classical integral transform to generalized functions.

Different definitions can be used according to what properties the generalized transform is required to have, such as inversion formulas, uniqueness, ... etc. However, there seem to be two main techniques to achieve such an extension. In one technique, the kernel of the integral transform is embedded in a suitably chosen testing-function space, while in the other one has to find a space of $C^{\infty}$-functions that is invariant under the integral transform and then apply the method of the adjoint that was first used by Schwartz to extend the Fourier transform to distributions.

No matter what one's definition is, one should always try to preserve the following property: if $f(t)$ is a suitably restricted conventional function for which both the conventional integral transform $\mathscr{F}_{c}(f)$ and the generalized integral transform $\mathscr{F}_{g}(f)$ exist, then they should be equal. By a generalized integral transform we mean an integral transform of a generalized function. Neither the definition of a generalized integral transform, nor the class of generalized functions to which it can be extended is universal. Our definition of the generalized integral transform is by no means the most general, but it is general enough to cover the applications. The technique we shall use is similar to the one used by Zemanian for Laplace transform [20].

First, let us assume that the generalized function $f$ belongs to at least one of the spaces $\mathcal{L}_{a, b}^{*}$ with $a<b$. Then, for each such $f$, define $\lambda_{f}=\{\sigma \mid$ there exist two real numbers $a_{\sigma}$ and $b_{\sigma}$ such that $a_{\sigma}<\sigma<b_{\sigma}$ and $\left.f \in \mathcal{E}_{a_{\sigma}, b_{\sigma}}^{*}\right\}$. It is readily seen that $\left(a_{\sigma}, b_{\sigma}\right)$ is contained in $\lambda_{f}$. In fact, it is not hard to see that $\lambda_{f}$ is an open set consisting of the union of all such intervals. Let $\sigma_{1}=\inf \lambda_{f}($ possibly $-\infty)$ and $\sigma_{2}=\sup \lambda_{f}($ possibly $\infty)$ and let $\Omega_{f}$ denote the open strip $\left\{s=x+i y \mid \sigma_{1}<x<\sigma_{2}\right\}$ if $s$ is complex or the open interval $\left(\sigma_{1}, \sigma_{2}\right)$ if $s$ is real. Finally, let us assume that the kernel $K(s, t)$ of the integral transform $\mathscr{F}$ belongs to the space $\mathcal{L}_{a, b}$ for $s \in \Omega_{f}$. Now we can define the generalized integral transform $\mathscr{F}$ of $f$ as follows:

The generalized integral transform $\mathscr{F}$ of $f$ is a conventional function defined on $\Omega_{f}$ and is given by

$$
\mathscr{F}_{g}(f)(s)=F_{g}(s)=\langle f(t), K(s, t)\rangle, \quad s \in \Omega_{f} .
$$

We call $\Omega_{f}$ the region of definition of $\mathscr{F}_{g}(f)$ and $\sigma_{1}$ and $\sigma_{2}$ the abscissas of definition.

5.1. If, in addition to the previous assumptions, the seminorms $\left\{\gamma_{k}\right\}_{k=1}^{\infty}$ have the property that

$$
\gamma_{k}(\psi \phi)=\sum_{j=0}^{k} P_{j}(\psi) \gamma_{j}(\phi)
$$

where $\psi$ is a $C^{\infty}$-function on $I, \phi \in \mathcal{L}_{a, b}$ and $P_{j}(t)$ are bounded functions of $t$, then arguments similar to those used in [20] show that $f$ can be extended so that $f \in \mathcal{L}^{*}\left(\sigma_{1}, \sigma_{2}\right)$. Moreover, $f \in \mathcal{L}^{*}(w, z)$ if either $w<\sigma_{1}$ or $z>\sigma_{2}$.

In this case, we require that $K(s, t) \in \mathcal{L}\left(\sigma_{1}, \sigma_{2}\right)$ for $s \in \Omega_{f}$ and definition (5.1) 
remains valid. It should be borne in mind that the operator $R$ as well as the seminorms $\left\{\gamma_{k}\right\}_{k=1}^{\infty}$ are tailored in such a way that the kernel function $K(s, t)$ is indeed in $\mathcal{L}_{a, b}$ or in $\mathcal{L}\left(\sigma_{1}, \sigma_{2}\right)$.

5.2. If $f(t)$ is a locally integrable function on $I$ such that $f(t) / \eta(t, a, b)$ is absolutely integrable on $I$, then both the conventional and the generalized integral transforms exist and they are equal as conventional functions in $\Omega_{f}$. For, by Lemma 3.1, $f(t)$ defines a regular functional on $\mathcal{L}_{a, b}$ and hence,

$$
\mathscr{F}_{g}(f)(s)=\langle f(t), K(s, t)\rangle=\int_{I} f(t) K(s, t) d t=\mathscr{F}_{c}(f)(s)
$$

for $s \in \Omega_{f}$.

LEMMa 5.1. Assume that

$$
R_{t} K(s, t)=P(s) K(s, t)
$$

where $P(s)$ is a polynomial in $s$ and $R_{t}$ means the operator $R$ acting on $K(s, t)$ as $a$ function of $t$. Then,

(i)

$$
\mathscr{F}_{g}\left(\left(R_{g}^{*}\right)^{k} f\right)(s)=P^{k}(s) \mathscr{F}_{g}(f)(s)
$$

for any nonnegative integer $k$.

(ii) $\left|\mathscr{F}_{g}(f)(s)\right| \leqslant G(|s|)$ for $\sigma_{1}<a \leqslant \operatorname{Re} s \leqslant b<\sigma_{2}$ where $G$ is a polynomial that depends in general on $a$ and $b$. In particular, if $\sigma_{2}=\infty$, then $\mathscr{F}_{g}(f)(s)$ is bounded by $a$ polynomial at $\infty$.

Proof. (i) First, observe that $R^{*}$ is continuous on $\mathcal{L}^{*}\left(\sigma_{1}, \sigma_{2}\right)$ (see equations (4.1) and (4.2)). Hence,

$$
\begin{aligned}
\left(\mathscr{F}_{g}\left(R_{g}^{*}\right)^{k} f\right)(s) & =\left\langle\left(R_{g}^{*}\right)^{k} f(t), K(s, t)\right\rangle=\left\langle f(t), R_{c}^{k} K(s, t)\right\rangle \\
& =P^{k}(s)\langle f(t), K(s, t)\rangle=P^{k}(s) \mathscr{F}_{g}(f)(s) .
\end{aligned}
$$

(ii) Since $f$ is a continuous linear functional on $\mathcal{L}_{a, b}$ for any $a$ and $b$ such that $\sigma_{1}<a \leqslant b<\sigma_{2}$, then there exist a constant $C$ and a nonnegative integer $m$ such that

$$
\begin{aligned}
\left|\mathscr{F}_{g}(f)(s)\right| & =|\langle f(t), K(s, t)\rangle| \leqslant C \max _{0 \leqslant k \leqslant m} \gamma_{k, a, b}(K(s, t)) \\
& \leqslant C \max _{0 \leqslant k \leqslant m} \sup _{t \in I}\left|\eta(t, a, b) R^{k} K(s, t)\right| \\
& =C \max _{0 \leqslant k \leqslant m}\left|P^{k}(s)\right| \sup _{t \in I}|\eta(t, a, b) K(s, t)| \\
& =C \max _{0 \leqslant k \leqslant m}|P(s)|^{k} \gamma_{0, a, b}(K(s, t)) \leqslant G(|s|) .
\end{aligned}
$$

Q.E.D.

We close this section by giving some examples of integral transforms that are special cases of our formulation. We shall state the interval $I$, the functions $\eta$, the operator $R$ and the space to which the kernel of the integral transform belongs.

It should be pointed out that in some of these examples, the seminorms are not exactly the same as those used in [21] but they are equivalent to them. 
1. (a) The two-sided Laplace transform. The conventional two-sided Laplace transform of $f(t)$ is defined by

$$
F(s)=\int_{-\infty}^{\infty} f(t) e^{-s t} d t
$$

$I=(-\infty, \infty), R=d / d t$ and

$$
\eta(t, a, b)= \begin{cases}e^{a t}, & 0 \leqslant t<\infty \\ e^{b t}, & -\infty<t<0\end{cases}
$$

where $a$ and $b$ are real numbers.

$K(s, t)=e^{-s t} \in \mathcal{L}\left(\sigma_{1}, \sigma_{2}\right)$ or equivalently $e^{-s t} \in \mathcal{L}_{a, b}$ for any $a$ and $b$ such that $\sigma_{1}<a \leqslant \operatorname{Re} s<b \leqslant \sigma_{2}$.

(b) The right-sided Laplace transform. This is defined by

$$
F(s)=\int_{T}^{\infty} f(t) e^{-s t} d t
$$

$I=(T, \infty), R=d / d t, \eta(t, a)=e^{a t}$ for $0 \leqslant t<\infty$ and zero otherwise. $e^{-s t} \in \mathcal{L}\left(\sigma_{1}\right)$ or equivalently $e^{-s t} \in \mathcal{L}_{a}$ for any $a$ such that $\sigma_{1}<a \leqslant \operatorname{Re} s$.

In both the above-mentioned cases, we have

$$
R_{t} K(s, t)=d e^{-s t} / d t=(-s) K(s, t)
$$

2. The Mellin transform. The conventional Mellin transform is defined by

$$
F(s)=\int_{0}^{\infty} f(t) t^{s-1} d t
$$

$I=(0, \infty), R=t d / d t$,

$$
\eta(t, a, b)= \begin{cases}t^{-a}, & 0<t \leqslant 1, \\ t^{-b}, & 1<t \leqslant \infty,\end{cases}
$$

for any real numbers $a$ and $b$. Also, $t^{s-1} \in \mathcal{L}\left(\sigma_{1}, \sigma_{2}\right)$ or equivalently $t^{s-1} \in \mathcal{L}_{a, b}$ for any $a$ and $b$ such that $\sigma_{1}<a \leqslant \operatorname{Re} s \leqslant b<\sigma_{2}$.

Finally, $R K(s, t)=t d t^{s-1} / d t=(s-1) t^{s-1}$.

3. The Stieltjes transform. The conventional Stieltjes transform is defined by

$$
F(s)=\int_{0}^{\infty} \frac{f(t)}{s+t} d t, \quad 0<s<\infty,
$$

$I=(0, \infty), R=t d / d t$,

$$
\eta(t, a, b)= \begin{cases}t^{a+1 / 2} & \text { for } 0<t<1 \\ t^{b+1 / 2} & \text { for } 1 \leqslant t<\infty\end{cases}
$$

and $(s+t)^{-1} \in \mathcal{L}_{a, b}$ for $a>-\frac{1}{2}$ and $b<\frac{1}{2}$. 
4. The Weierstrass transform. The conventional Weierstrass transform of $f(t)$ is defined by

$$
F(s)=\frac{1}{\sqrt{4 \pi}} \int_{-\infty}^{\infty} f(t) \exp \left[-(s-t)^{2} / 4\right] d t
$$

$I=(-\infty, \infty), R=d / d t$

$$
\eta(t, a, b)= \begin{cases}\exp \left(t^{2} / 4-a t / 2\right), & -\infty<t<0 \\ \exp \left(t^{2} / 4-b t / 2\right), & 0 \leqslant t<\infty\end{cases}
$$

and $\exp \left[-(s-t)^{2} / 4\right] \in \mathcal{L}\left(\sigma_{1}, \sigma_{2}\right)$ or equivalently $\exp \left[-(s-t)^{2} / 4\right] \in \mathcal{L}_{a, b}$ if $\sigma_{1}<a$ $\leqslant \operatorname{Re} s \leqslant b<\sigma_{2}$.

Moreover, $(d / d t) \exp \left[-(s-t)^{2} / 4\right]=((s-t) / 2) \exp \left[-(s-t)^{2} / 4\right]$.

5. The Airy transform. The conventional Airy transform is defined by

$$
F(s)=\int_{0}^{\infty} \operatorname{Ai}(s t) f(t) d t, \quad 0<s<\infty,
$$

where $\operatorname{Ai}(x)$ is the Airy function defined by (see [13])

$$
\operatorname{Ai}(x)=\frac{1}{\pi} \int_{0}^{\infty} \cos \left(1 / 3 t^{3}+x t\right) d t
$$

The space $\varrho_{a, b}$ of this transform has not been explicitly discussed in the literature. However, it is not hard to see that $I=(0, \infty), R=\frac{1}{t} d^{2} / d t^{2}, \eta(t, a)=e^{2 / 3 a t^{3 / 2}}$, $\operatorname{Ai}(s t) \in \mathcal{L}\left(\sigma_{1}\right)$ if $s>a>\sigma_{1}$ and $R \operatorname{Ai}(s t)=s^{3} \operatorname{Ai}(s t)$.

6. The Hankel transform. The Hankel transform of $f(t)$ is defined by

$$
F(s)=\int_{0}^{\infty} f(t) \sqrt{s t} J_{\mu}(s t) d t, \quad 0<s<\infty,
$$

where $J_{\mu}(x)$ is the Bessel function of the first kind and order $\mu, I=(0, \infty)$,

$$
R=t^{-\mu-1 / 2} \frac{d}{d t} t^{2 \mu+1} \frac{d}{d t} t^{-\mu-1 / 2}=\frac{d^{2}}{d t^{2}}-\frac{\left(\mu^{2}-1 / 4\right)}{t^{2}},
$$

$\eta(t, a, \mu)=e^{-a t} t^{-\mu-1 / 2}$ where $a$ is a positive real number and $\mu>-\frac{1}{2}$.

We have $\sqrt{s t} J_{\mu}(s t) \in \mathcal{L}_{a, \mu}$ for any positive number $a$ and $\mu>-\frac{1}{2}$. Moreover, $R \sqrt{s t} J_{\mu}(s t)=-s^{2} \sqrt{s t} J_{\mu}(s t)$.

7. The $K$ transform. The conventional $K$ transform is defined by

$$
F(s)=\int_{0}^{\infty} f(t) \sqrt{s t} K_{\mu}(s t) d t
$$

where $K_{\mu}(t)$ is the modified Bessel function of the third kind and order $\mu$, $I=(0, \infty)$,

$$
R=t^{-\mu-1 / 2} \frac{d}{d t} t^{2 \mu+1} \frac{d}{d t} t^{-\mu-1 / 2}=\frac{d^{2}}{d t^{2}}-\frac{4 \mu^{2}-1}{4 t^{2}},
$$

$\eta(t, a, \mu)=e^{a t} j_{\mu}(t)$ for $0<t<\infty$, where

$$
j_{\mu}(t)=\left\{\begin{array}{ll}
t^{\mu-1 / 2}, & \mu>0, \\
{[\sqrt{t} h(t)]^{-1},} & \mu=0,
\end{array} \text { and } \quad h(t)= \begin{cases}\ln t, & 0<t<\frac{1}{e}, \\
-1, & \frac{1}{e} \leqslant t<\infty .\end{cases}\right.
$$


For every fixed $s(s \neq 0)$ we have $\sqrt{s t} K_{\mu}(s t) \in \mathcal{L}_{a, \mu}$ and $R \sqrt{s t} K_{\mu}(s t)=s^{2} \sqrt{s t} K_{\mu}(s t)$.

6. Asymptotic expansions. The formulation of the generalized integral transforms outlined in the preceding section together with the technique devised by Wong [18] for the Fourier transform will now be combined to derive asymptotic expansions for some other integral transforms.

In what follows, we shall be concerned primarily with a class of integral transforms called the class of $h$ transforms. This class was studied in great depth and was given its name by Bleistein and Handelsman [1].

An integral transform is called an $h$ transform if its kernel function $K(s, t)$ is of the form $K(s t)$, i.e.

$$
\mathscr{F}(f)(s)=\int_{I} f(t) K(s t) d t
$$

Since we are interested in the behavior of $\mathscr{F}(f)(s)$ as $s \rightarrow \infty$, it is very reasonable to assume that $\sigma_{2}=\infty$ so that $\Omega_{f}$ is of the form $\left\{s \mid \operatorname{Re} s>\sigma_{1}\right\}$ if $s$ is complex or of the form $\left(\sigma_{1}, \infty\right)$ if $s$ is real. Throughout this section, we assume that $I=(0, \infty)$ and the kernel $K(s t)$ satisfies the following conditions:

$\left(\mathrm{A}_{1}\right) K(s t)$ is in some testing function space $\mathcal{L}\left(\sigma_{1}\right)$ for some real number $\sigma_{1}$.

$\left(\mathrm{A}_{2}\right) R K(s t)=P(s) K(s t)$ where $R$ is a differential operator of the form (3.1) and $P(s)$ is a polynomial in $s$ of degree $\gamma \geqslant 1$ and does not have any zeros in $\operatorname{Re} s \geqslant 0$.

$\left(\mathrm{A}_{3}\right) K(0)$ is finite and $K(s) \rightarrow 0$ as $s \rightarrow \infty$ in $0 \leqslant|\arg s|<\frac{\pi}{2}$.

Furthermore, we shall assume that

$\left(\mathrm{B}_{1}\right) t^{\beta}(\beta>-1)$ is in the space $\widehat{\iota^{*}}\left(\sigma_{1}\right)$ defined below.

$\left(\mathrm{B}_{2}\right) R^{*} t^{\beta}=O\left(t^{\beta-\gamma}\right)$ as $t \rightarrow 0+$.

$\left(\mathrm{B}_{3}\right)(\eta(t, a, b))^{-1}$ is bounded in $(0, \varepsilon]$ for some $\varepsilon>0$.

Finally, let us define the space $\widehat{\mathcal{L}^{*}}\left(\sigma_{1}\right)$ as follows: $f \in \widehat{\mathrm{L}^{*}}\left(\sigma_{1}\right)$ if and only if

(i) $f$ is locally integrable on $(0, \infty)$ and $f \in \mathcal{L}^{*}\left(\sigma_{1}\right)$.

(ii) $f$ has a conventional $\mathscr{F}$-transform $\mathscr{F}_{c}(f)(s)$ that converges uniformly for sufficiently large $s$.

(iii) $\mathscr{F}_{c}(f)=\mathscr{F}_{g}(f)$.

Certainly, if $f(t)$ satisfies the assumptions of remark 5.2, it must belong to $\widehat{L^{*}}\left(\sigma_{1}\right)$.

THEOREM 6.1. Let $f(t)$ be a function of the positive real variable $t$ satisfying the following conditions:

$\left(\mathrm{C}_{1}\right)$ As $t \rightarrow 0+$

$$
f(t) \sim \sum_{k=0}^{\infty} a_{k} t^{(k+\lambda-\alpha) / \alpha}
$$

where $\lambda>0$ and $\alpha>0$. Moreover, the expansion (6.1) is $\gamma m$-times differentiable where $m$ is a nonnegative integer.

$\left(\mathrm{C}_{2}\right)\left(R_{c}^{*}\right)^{j} f$ is a member of the space $\widehat{\mathcal{L}^{*}}\left(\sigma_{1}\right)$ for $j=0,1,2, \ldots, \gamma$. Let $n$ be a positive integer satisfying

$$
\gamma m<(n+\lambda) / \alpha \leqslant \gamma m+1
$$


Then, for sufficiently large s we have

$$
\mathscr{F}_{c}(f)(s)=\int_{0}^{\infty} f(t) K(s t) d t=\sum_{k=0}^{n-1} a_{k} \mathscr{F}_{c}\left(t^{(k+\lambda-\alpha) / \alpha}\right)(s)+\delta_{m, n}(s)
$$

where $\delta_{m, n}(s)=o\left(|s|^{-\gamma m}\right)$ as $s \rightarrow \infty$, where $s \rightarrow \infty$ in the sector $0 \leqslant|\arg s|<\frac{\pi}{2}$.

Proof. First of all, note that the integral in (6.3) exists because of $\left(C_{2}\right)$ (at least for $\left.\operatorname{Re} s>\sigma_{1}\right)$. Set

$$
f(t)=\sum_{k=0}^{n-1} a_{k} t^{(k+\lambda-\alpha) / \alpha}+\phi_{n}(t) \text { for } n=1,2, \ldots
$$

Because of $\left(C_{2}\right)$ and $\left(B_{1}\right)$, it follows that each function in equation (6.4) defines a generalized function on $\mathcal{L}\left(\sigma_{1}\right)$.

Taking the generalized $\mathscr{F}$ transform of both sides of (6.4) yields

$$
\mathscr{F}_{g}(f)(s)=\sum_{k=0}^{n-1} a_{k} \mathscr{F}_{g}\left(t^{(k+\lambda-\alpha) / \alpha}\right)(s)+\mathscr{F}_{g}\left(\phi_{n}\right)(s) .
$$

The operator $R^{*}$ is continuous on $\mathcal{L}^{*}\left(\sigma_{1}\right)$; hence by Lemma 5.1 we have

$$
\mathscr{F}_{g}\left(\phi_{n}\right)(s)=\frac{1}{P^{m}(s)} \mathscr{F}_{g}\left(R_{g}^{* m} \phi_{n}\right)(s)
$$

From equation (6.4), $\left(\mathrm{C}_{1}\right)$ and $\left(\mathrm{B}_{2}\right)$, we obtain that

$$
\left(R_{c}^{*}\right)^{j} \phi_{n}(t)=O\left(t^{(n+\lambda-\alpha) / \alpha-\gamma j}\right) \quad \text { as } t \rightarrow 0+, \text { for } j=0,1, \ldots, m .
$$

With the aid of equation (6.7), $\left(\mathrm{A}_{3}\right)$ and remark 4.1 one can easily show that

$$
\mathscr{F}_{g}\left(R_{g}^{* m} \phi_{n}\right)=\mathscr{F}_{g}\left(R_{c}^{* m} \phi_{n}\right) \text {. }
$$

Therefore, throughout the rest of the proof, we shall drop the subscript. Thus, (6.6) becomes

$$
\mathscr{F}_{g}\left(\phi_{n}\right)(s)=\frac{1}{P^{m}(s)} \mathscr{F}_{g}\left(R^{* m} \phi_{n}\right)(s)
$$

Upon inserting (6.9) into (6.5) using $\left(C_{2}\right)$ and $\left(B_{1}\right)$ we get

$$
\mathscr{F}_{c}(f)(s)=\int_{0}^{\infty} f(t) K(s t) d t=\sum_{k=0}^{n-1} a_{k} \mathscr{F}_{c}\left(t^{(k-\lambda-\alpha) / \alpha}\right)(s)+\delta_{m, n}(s)
$$

where $\delta_{m, n}(s)=\left(P^{m}(s)\right)^{-1} \mathscr{F}_{g}\left(R^{* m} \phi_{n}\right)(s)$.

Since each term in (6.10) defines a continuous function in some half-plane of the form $\operatorname{Re} s>d$ for some $d>0$ (see also $\left(\mathrm{A}_{2}\right)$ ), it follows that (6.10) holds not only in the sense of generalized functions, but also as conventional functions.

Since the degree of $P(s)$ is $\gamma$, the proof will be completed if we can show that $\mathscr{F}_{g}\left(R^{* m} \phi_{n}\right)(s) \rightarrow 0$ as $s \rightarrow \infty$.

It suffices to show that $R^{* m} \phi_{n}$ is in $\widehat{L^{*}}\left(\sigma_{1}\right)$. For in this case, we shall have

$$
\mathscr{F}_{g}\left(R^{* m} \phi_{n}\right)(s)=\mathscr{F}_{c}\left(R^{* m} \phi_{n}\right)(s)=\int_{0}^{\infty} R^{* m} \phi_{n}(t) K(s t) d t
$$

where the integral converges uniformly for sufficiently large $s$. Thus, for any $\varepsilon>0$, 
there exist $s_{0}$ and $c>0$ such that $\left|\int_{c}^{\infty} R^{* m} \phi_{n}(t) K(s t) d t\right|<\varepsilon / 2$ for all $s>s_{0}>0$.

On the other hand, by the Lebesgue dominated convergence theorem and the fact that $K(s t) \rightarrow 0$ as $s \rightarrow \infty$, we have

$$
\left|\int_{0}^{c} R^{* m} \phi_{n}(t) K(s t) d t\right|<\varepsilon / 2 \text { for sufficiently large } s .
$$

Now, to show that $R^{* m} \phi_{n}$ is in $\widehat{\mathrm{C}^{*}}\left(\sigma_{1}\right)$, we write

$$
R^{* m} \phi_{n}(t)=R^{* m} \phi_{n}(t) \chi_{(0 \xi]}+R^{* m} \phi_{n}(t) \chi_{[\varepsilon, \infty)}=g_{1}(t)+g_{2}(t)
$$

for some $\varepsilon>0$, where $\chi$ is the characteristic function of the indicated interval.

Applying $R^{* m}$ to $(6.4)$, using $\left(\mathrm{C}_{2}\right)$ and $\left(\mathrm{B}_{1}\right)$, one can show that $g_{2}(t)$ is indeed in $\widehat{E^{*}}\left(\sigma_{1}\right)$.

As for $g_{1}(t)$, we have

$$
\begin{aligned}
\left|\int_{0}^{\infty} g_{1}(t) \psi(t) d t\right| & =\left|\int_{0}^{\varepsilon} R^{* m} \phi_{n}(t) \psi(t) d t\right|=\left|\int_{0}^{\varepsilon} \frac{R^{* m} \phi_{n}(t)}{\eta(t)} \eta(t) \psi(t) d t\right| \\
& \leqslant \gamma_{0}(\psi) \int_{0}^{\varepsilon} \frac{R^{* m} \phi_{n}(t)}{\eta(t)} \mid d t<\infty \quad \text { for any } \psi(t) \in \mathcal{L}\left(\sigma_{1}\right) .
\end{aligned}
$$

The last inequality follows from (6.7) (for $j=m$ ) and $\left(\mathrm{B}_{3}\right)$. Thus, $g_{1}(t)$ is also in $\widehat{\mathcal{L}^{*}}\left(\sigma_{1}\right)$ and consequently so is $R^{* m} \phi_{n}$. Q.E.D.

Corollary 6.1 (LAPlaCE TRANSFORM). Let $f(t)$ satisfy conditions $\left(\mathrm{C}_{1}\right)$ and $\left(\mathrm{C}_{2}\right)$ where $\mathcal{L}\left(\sigma_{1}\right)$ is defined in Example 1(b) of $\$ 5$.

Let $n$ be a positive integer satisfying $m<(n+\lambda) / \alpha \leqslant m+1$.

Then for sufficiently large $s$, we have

$$
\int_{0}^{\infty} f(t) e^{-s t} d t=\sum_{k=0}^{n-1} \Gamma\left(\frac{k+\lambda}{\alpha}\right) \frac{a_{k}}{s^{(k+\lambda) / \alpha}}+\delta_{m, n}(s)
$$

where $\delta_{m, n}(s)=o\left(|s|^{-m}\right)$ as $s \rightarrow \infty($ see $[13])$.

COROllary 6.2 (THE AIRY TRANSFORM). Let $f(t)$ satisfy conditions $\left(\mathrm{C}_{1}\right)$ and $\left(\mathrm{C}_{2}\right)$ where $\mathcal{L}\left(\sigma_{1}\right)$ is defined in Example 5 of $\$ 5$. Let $n$ be a positive integer satisfying $3 m<(n+\lambda) / \alpha \leqslant 3 m+1$.

Then, for sufficiently large $s$, we have

$$
\int_{0}^{\infty} f(t) \operatorname{Ai}(s t) d t=\sum_{k=0}^{n-1} \frac{a_{k} 3^{(2(k+\lambda) / 3 \alpha)-7 / 6}}{2 \pi s^{(k+\lambda) / \alpha}} \Gamma\left(\frac{k+\lambda}{3 \alpha}\right) \Gamma\left(\frac{k+\lambda+\alpha}{3 \alpha}\right)+\delta_{m, n}(s)
$$

where $\delta_{m, n}(s)=o\left(|s|^{-3 m}\right)$ as $s \rightarrow \infty($ see $[1])$.

Corollary 6.3 (THE $K_{\mu}$ TRANSFORM, $\mu \geqslant-\frac{1}{2}$ ). Let $f(t)$ satisfy $\left(\mathrm{C}_{1}\right)$ and $\left(\mathrm{C}_{2}\right)$ with $\alpha=1$ and $\lambda+\frac{1}{2}>\mu$.

Let $n$ be a positive integer satisfying

$$
2 m<\underline{n}+\lambda-\mu+\frac{1}{2} \leqslant 2 m+1 .
$$


Then, for sufficiently large s, we have

$$
\begin{aligned}
& \int_{0}^{\infty} f(t) \sqrt{s t} K_{\mu}(s t) d t \\
& \quad=\sum_{k=0}^{n-1} 2^{k+\lambda-3 / 2} \Gamma\left(\frac{k+\lambda+\mu+1 / 2}{2}\right) \Gamma\left(\frac{k+\lambda-\mu+1 / 2}{2}\right) \frac{a_{k}}{s^{(k+\lambda)}}+\delta_{m, n}(s)
\end{aligned}
$$

where $\delta_{m, n}(s)=o\left(|s|^{-2 m}\right)$ as $s \rightarrow \infty($ see [1] $)$.

Note that condition $\left(B_{3}\right)$ is violated, i.e., $\eta(t)^{-1}=t^{-\mu+1 / 2}$ is not necessarily bounded in a neighborhood of zero. Nevertheless, the proof remains valid if $n$ satisfies (6.11) instead of (6.2).

7. The Hankel transform. Wong's definition of the Hankel transform [16] is slightly different from Zemanian's [21]. However, the latter seems more appropriate for our purpose and we shall adopt it.

Unless otherwise stated, the space $\mathcal{L}_{a, \mu}$ will stand for the space $\mathcal{L}_{a, \mu}$ of Example 6; $\mu$ will be restricted so that $\mu \geqslant-\frac{1}{2}$ and $s$ will be a nonnegative real number.

It should be emphasized that the technique we used for the $K_{\mu}$ transform is no longer applicable because conditions $\left(A_{3}\right)$ and $\left(B_{1}\right)$ are violated.

Although Zemanian has given two different definitions of the generalized Hankel transform (cf. [7] and [21]) neither one seems very suitable for our use. His definition in [7] does not admit a generalized Hankel transform for the function $t^{n}(n>0)$ and even though his definition in [21] admits such a transform, it does not lead directly to the results obtained by Wong [16]. Therefore, we shall construct a new testingfunction space that will enable us to define a generalized Hankel transform for $t^{n+\lambda-1}$, for any nonnegative integer $n$ and any $\lambda$ such that $\operatorname{Re}(\lambda+1 / 2+\mu)>0$, that will agree with Wong's results.

Let us introduce the space $S_{\mu}$ as follows:

A function $\phi(t)$ is in $S_{\mu}$ if and only if it is a $C^{\infty}$-function on $I=(0, \infty)$ and for each pair of nonnegative integers $m$ and $k$

$$
\gamma_{m, k}^{\mu}(\phi)=\sup _{t \in I}\left|t^{m}(t D)^{k} t^{-\mu-1 / 2} \phi(t)\right|<\infty .
$$

$S_{\mu}$ is a linear space and $\left\{\gamma_{m, k}^{\mu}\right\}_{m, k=0}^{\infty}$ is a countable family of separating seminorms. We list some properties of the space $S_{\mu}$ and refer the reader to [21] for the proofs.

(1) If $\phi(t) \in S_{\mu}$, then $\phi(t)=O\left(t^{\mu+1 / 2}\right)$ as $t \rightarrow 0+$ and, for each nonnegative integer $k, D^{k} \phi(t)$ tends to zero faster than any power of $\frac{1}{t}$ as $t \rightarrow \infty$.

(2) $S_{\mu}$ is a Fréchet space.

(3) If $q$ is an even integer, then $S_{\mu+q} \subset S_{\mu}$ and the topology of $S_{\mu+q}$ is stronger than the topology induced on it by $S_{\mu}$.

(4) For $\mu \geqslant-\frac{1}{2}$, the conventional Hankel transform is an automorphism on $S_{\mu}$.

(5) The operator $R=d^{2} / d t^{2}-\left(4 \mu^{2}-1\right) / 4 t^{2}$ is continuous on $S_{\mu}$.

Now define the space $S^{\mu}$ by $S^{\mu}=\cup_{q=0}^{\infty} S_{\mu+2 q}$. The topology of $S^{\mu}$ is the projective limit topology [8], i.e. the weakest topology on $S^{\mu}$ for which all the mappings $S^{\mu} \rightarrow S_{\mu+2 q}$ are continuous. The following properties of $S^{\mu}$ can be easily proved:

(1) If $\phi(t) \in S^{\mu}$, then $\phi(t)=O\left(t^{\mu+2 q}\right)$ as $t \rightarrow 0+$ for any nonnegative integer $q$ and $D^{q} \phi(t)$ tends to zero faster than any power of $\frac{1}{t}$ as $t \rightarrow \infty$. 
(2) $S^{\mu}$ is a Fréchet space.

(3) For $\mu \geqslant-\frac{1}{2}$, the conventional Hankel transform is an automorphism on $S^{\mu}$.

(4) The operator $R$ is continuous on $S^{\mu}$.

(5) The function $t^{n}$ is in the dual space $\left(S^{\mu}\right)^{*}$ of $S^{\mu}$ for any integer $n$.

We define the generalized Hankel transform $\mathscr{F}_{g}$ for any member $f$ of the space $\left(S^{\mu}\right)^{*}$ by the formula

$$
\left\langle\mathscr{F}_{g}(f), \phi\right\rangle=\left\langle f, \mathscr{F}_{c}(\phi)\right\rangle
$$

where $f \in\left(S^{\mu}\right)^{*}$ and $\phi$ traverses $S^{\mu}$.

7.1. This definition makes sense since $\mathscr{F}_{c}$ is an automorphism on $S^{\mu}$ and from this it follows also that $\mathscr{F}_{g}$ is an automorphism on $\left(S^{\mu}\right)^{*}$. Moreover, this definition agrees with our previous definition of the generalized Hankel transform as an application of the generalized function $f$ to the kernel function $\sqrt{s t} J_{\mu}(s t)$ whenever $f \in \mathcal{L}_{a, \mu}^{*}$, i.e. $\left\langle\left\langle f(t), \sqrt{s t} J_{\mu}(s t)\right\rangle, \phi(s)\right\rangle=\left\langle\mathscr{F}_{g}(f)(s), \phi(s)\right\rangle=\left\langle f(t), \mathscr{F}_{c}(\phi)(t)\right\rangle$ for all $\phi(s) \in S^{\mu}$.

This can be seen from the fact that $\mathcal{L}_{a, \mu}^{*} \subset\left(S_{\mu}\right)^{*} \subset\left(S^{\mu}\right)^{*}$ and from Theorem 3 in [7].

7.2. If $f(t)$ is a locally integrable function on $(0, \infty)$ such that $f \in L^{1}(\varepsilon, \infty)$ for any $\varepsilon>0$ and $f(t)=O\left(t^{-\mu-1 / 2-\alpha}\right)$ as $t \rightarrow 0+$ for $\alpha<1$, then it is easy to see that $f \notin \mathcal{L}_{a, \mu}^{*}$ but $f \in\left(S^{\mu}\right)^{*}$ and that $f$ has a conventional Hankel transform $\mathscr{F}_{c}(f)$ that is equal to $\mathscr{F}_{g}(f)$ as functionals on $S^{\mu}$.

For

$$
\begin{aligned}
\left\langle\mathscr{F}_{g}(f), \phi\right\rangle & =\left\langle f, \mathscr{F}_{c}(\phi)\right\rangle=\int_{0}^{\infty} f(t) \int_{0}^{\infty} \phi(s) \sqrt{s t} J_{\mu}(s t) d s d t \\
& =\int_{0}^{\infty} \phi(s) \int_{0}^{\infty} f(t) \sqrt{s t} J_{\mu}(s t) d t d s=\int_{0}^{\infty} \mathscr{F}_{c}(f)(s) \phi(s) d s \\
& =\left\langle\mathscr{F}_{c}(f), \phi\right\rangle \text { for all } \phi \in S^{\mu} .
\end{aligned}
$$

Interchanging the integrals is permissible by Fubini's theorem.

7.3. In addition to that, if both $\mathscr{F}_{c}(f)(s)$ and $\mathscr{F}_{g}(f)(s)$ define continuous functions in some subinterval $J$ of $\Omega_{f}=\left(\sigma_{1}, \infty\right)$, then they must be equal as functions on $J$. This follows from the fact that $\mathscr{D}(I) \subset \varrho_{a, \mu}$ and from standard arguments in the theory of generalized functions [3].

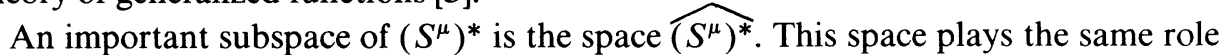
as the space $\widehat{\mathrm{L}_{a, b}^{*}}$ of $\S 6$ and is defined analogously.

Now we are able to define the Hankel transform of the functions $t^{\beta-1}$ for $\operatorname{Re}(\beta+\mu+1 / 2)>0$.

LEMMA 7.1. (1)If $\left\{f_{n}\right\}$ is a sequence in $\widehat{\left(S^{\mu}\right)^{*}}$ that converges to zero in $\left(S^{\mu}\right)^{*}$, then $\lim _{n \rightarrow \infty} \mathscr{F}_{c}\left(f_{n}\right)=\lim _{n \rightarrow \infty} \mathscr{F}_{g}\left(f_{n}\right)=0$ in the sense of $\left(S^{\mu}\right)^{*}$.

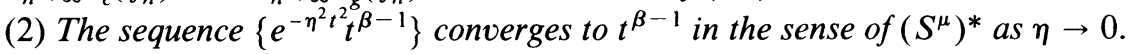

$$
\begin{aligned}
& \lim _{\eta \rightarrow 0} \mathscr{F}_{g}\left(e^{-\eta^{2} t^{2}} t^{\beta-1}\right)=\lim _{\eta \rightarrow 0} \int_{0}^{\infty} e^{-\eta^{2} t^{2}} t^{\beta-1} \sqrt{s t} J_{\mu}(s t) d t \\
& =\mathscr{F}_{g}\left(t^{\beta-1}\right)=\frac{\Gamma\left(\frac{\beta}{2}+\frac{\mu}{2}+\frac{1}{4}\right) 2^{\beta-1 / 2}}{\Gamma\left(\frac{\mu}{2}-\frac{\beta}{2}+\frac{3}{4}\right) s^{\beta}}
\end{aligned}
$$

where the convergence is understood to be in the sense of $\left(S^{\mu}\right)^{*}$. 
Proof. (1) Since $\mathscr{F}_{g}$ is an automorphism on $\left(S^{\mu}\right)^{*}$, the result follows immediately.

(2) For any $\phi(t) \stackrel{g}{\in} S^{\mu}$, we have $\int_{0}^{\infty}\left|t^{\beta-1} \phi(t)\right| d t<\infty$. Hence, by the Lebesgue dominated convergence theorem, it follows that

$$
\lim _{\eta \rightarrow 0}\left(\left\langle e^{-\eta^{2} t^{2}} t^{\beta-1}, \phi(t)\right\rangle-\left\langle t^{\beta-1}, \phi(t)\right\rangle\right)=\lim _{\eta \rightarrow 0} \int_{0}^{\infty}\left(1-e^{-\eta^{2} t^{2}}\right) t^{\beta-1} \phi d t=0 .
$$

(3) The sequence $\left\{e^{-\eta^{2} t^{2} t^{\beta-1}}\right\}$ is in $\widehat{\left(S^{\mu}\right)^{*}}$. Therefore,

$$
\begin{aligned}
\lim _{\eta \rightarrow 0} \mathscr{F}_{g}\left(e^{-\eta^{2} t^{2}} t^{\beta-1}\right)(s) & =\lim _{\eta \rightarrow 0}\left\langle e^{-\eta^{2} t^{2} t^{\beta-1}}, \sqrt{s t} J_{\mu}(s t)\right\rangle \\
& =\lim _{\eta \rightarrow 0} \int_{0}^{\infty} e^{-\eta^{2} t^{2} t^{\beta-1}} \sqrt{s t} J_{\mu}(s t) d t=\frac{\Gamma\left(\frac{\beta}{2}+\frac{\mu}{2}+\frac{1}{4}\right) 2^{\beta-1 / 2}}{\Gamma\left(\frac{\mu}{2}-\frac{\beta}{2}+\frac{3}{4}\right) s^{\beta}} \\
& =\mathscr{F}_{g}\left(t^{\beta-1}\right)(s) .
\end{aligned}
$$

The second equality from the right follows from Lemma 1 in [16]. Clearly, the definition of $\mathscr{F}_{g}\left(t^{\beta-1}\right)$ is independent of the representative sequence $\left\{e^{-\eta^{2} t^{2} t^{\beta-1}}\right\}$ by part (1).

THEOREM 7.1. Let $f(t)$ be defined on $(0, \infty)$ and satisfy the following conditions:

$\left(\mathrm{D}_{1}\right)$ As $t \rightarrow 0+$

$$
f(t) \sim \sum_{k=0}^{\infty} a_{k} t^{k+\lambda-1}
$$

where $a_{0} \neq 0, \operatorname{Re}(\lambda+\mu+1 / 2)>0$ and $\mu \geqslant 0$. Moreover, the expansion in (7.1) is $2 m$-times differentiable where $m$ is a nonnegative integer satisfying $2 m \geqslant \operatorname{Re}(\lambda+1 / 2)$.

$\left(\mathrm{D}_{2}\right) R^{j} f(t)$ is continous and belongs to $\left(S^{\mu}\right)^{*}$ for $j=0,1, \ldots, m$.

Let $n$ be a positive integer such that

$$
2 m-\operatorname{Re}(\lambda+1 / 2)<n \leqslant 2 m-\operatorname{Re}(\lambda-1 / 2) .
$$

Then, for sufficiently large $s$, we have

$$
\begin{aligned}
\mathscr{F}_{c}(f)(s) & =\int_{0}^{\infty} f(t) \sqrt{s t} J_{\mu}(s t) d t \\
& =\sum_{k=0}^{n-1} a_{k} \frac{\Gamma((k+\lambda+\mu) / 2+1 / 4) 2^{k+\lambda-1 / 2}}{\Gamma((\mu-k-\lambda) / 2+3 / 4) s^{k+\lambda}}+\delta_{m, n}(s)
\end{aligned}
$$

where $\delta_{m, n}(s)=o\left(1 / s^{2 m-1 / 2}\right)$ as $s \rightarrow \infty$.

Proof. For each $n>0$, set

$$
f(t)=\sum_{k=0}^{n-1} a_{k} t^{k+\lambda-1}+\phi_{n}(t), \quad 0<t<\infty .
$$

Since each term in (7.4) defines a functional on $S^{\mu}$, then taking the generalized Hankel transform of both sides of (7.4) yields

$$
\begin{aligned}
\mathscr{F}_{g}(f)(s) & =\int_{0}^{\infty} f(t) \sqrt{s t} J_{\mu}(s t) d t \\
& =\sum_{k=0}^{n-1} a_{k} \frac{\Gamma((k+\lambda+\mu) / 2+1 / 4) 2^{k+\lambda-1 / 2}}{\Gamma((\mu-k-\lambda) / 2+3 / 4) s^{k+\lambda}}+\mathscr{F}_{g}\left(\phi_{n}\right)(s) .
\end{aligned}
$$


The first equality follows from $\left(D_{2}\right)$ and the second from Lemma 7.1.

Since $R$ is selfadjoint and continuous on $S^{\mu}$, we have by Lemma 5.1 that

$$
\mathscr{F}_{g}\left(\phi_{n}\right)(s)=\mathscr{F}_{g}\left(R_{g}^{m} \phi_{n}\right)(s) / s^{2 m} \text {. }
$$

Upon inserting (7.6) in (7.5) one gets

$$
\int_{0}^{\infty} f(t) \sqrt{s t} J_{\mu}(s t) d t=\sum_{k=0}^{n-1} a_{k} \frac{\Gamma((k+\lambda+\mu) / 2+1 / 4) 2^{k+\lambda-1 / 2}}{\Gamma((\mu-k-\lambda) / 2+3 / 4) s^{k+\lambda}}+\delta_{m, n}(s)
$$

where

$$
\delta_{m, n}(s)=\mathscr{F}_{g}\left(R_{g}^{m} \phi_{n}\right)(s) / s^{2 m} .
$$

But from equations (7.1) and (7.4), we obtain

$$
R^{j} \phi_{n}(t)=O\left(t^{n+\lambda-1-2 j}\right) \quad \text { as } t \rightarrow 0+
$$

for $j=0,1, \ldots, m$. Once more, remark 4.1 and equation (7.9) show that $\mathscr{F}_{g}\left(R_{g}^{m} \phi_{n}\right)=$ $\mathscr{F}_{g}\left(R_{c}^{m} \phi_{n}\right)$. The subscript will be discarded in the remaining part of the proof.

It is easy to see that there are constants $C_{k}, k=0,1, \ldots, n-1$, such that

$$
R^{m} \phi_{n}(t)=R^{m} f(t)-\sum_{k=0}^{n-1} C_{k} t^{k+\lambda-1-2 m} .
$$

Then, according to $\left(\mathrm{D}_{2}\right),(7.2)$ and (7.10), we have

$$
\int_{\varepsilon}^{\infty}\left|R^{m} \phi_{n}(t) \sqrt{s t} J_{\mu}(s t)\right| d t<\infty
$$

We now turn to equation (7.9) and put $j=m$. This, together with equation (7.2) and $\left(D_{2}\right)$, shows that

$$
\int_{0}^{\varepsilon}\left|R^{m} \phi_{n}(t) \sqrt{s t} J_{\mu}(s t)\right| d t<\infty
$$

In fact, the same argument shows that $R^{m} \phi_{n} \in \widehat{\left(S^{\mu}\right)^{*}}$. Therefore, equation (7.8) becomes

$$
\delta_{m, n}(s)=\frac{1}{s^{2 m}} \int_{0}^{\infty} R^{m} \phi_{n}(t) \sqrt{s t} J_{\mu}(s t) d t=\frac{1}{s^{2 m-1 / 2}} \int_{0}^{\infty} R^{m} \phi_{n}(t) \sqrt{t} J_{\mu}(s t) d t .
$$

Now it is clear that every term in (7.7) defines a continuous function (at least for sufficiently large $s$ ). Thus, by remark 7.3, it follows that equation (7.7) holds not only in the sense of generalized functions, e.g. in $\left(S^{\mu}\right)^{*}$, but also as conventional functions.

Finally, the same argument used by Wong $[16, \S 4]$ shows that

$$
\int_{0}^{\infty} R^{m} \phi_{n}(t) \sqrt{t} J_{\mu}(s t) d t \rightarrow 0 \quad \text { as } s \rightarrow \infty .
$$

Hence, by equation (7.11), we obtain

$$
\delta_{m, n}(s)=o\left(\frac{1}{s^{2 m-1 / 2}}\right) \quad \text { as } s \rightarrow \infty .
$$

Q.E.D. 
In proving Theorem 7.1 we tried to adhere to the general procedure outlined in the previous sections. But as a result of that, Theorem 7.1 is not stated as generally as it could be. The restriction $\mu \geqslant 0$ can be relaxed to $\mu \geqslant-\frac{1}{2}$ and condition $\left(D_{2}\right)$ can be weakened to condition (iii) of $\S 4$ in [19], if one uses the operator

$$
M_{\mu}=x^{-\mu} \frac{d}{d x} x^{\mu}
$$

instead of the operator $R$.

\section{REFERENCES}

1. N. Bleistein and R. A. Handelsman, Asymptotic expansions of integrals, Holt, Rinehart and Winston, New York, 1975.

2. P. Durbin, Asymptotic expansion of Laplace transforms about the origin using generalized functions, $\mathrm{J}$. Inst. Math. Appl. 23 (1979), 181-192.

3. I. M. Gel'fand and G. E. Shilov, Generalized functions, vols. 1 and 2, Academic Press, New York, 1964.

4. R. A. Handelsman and J. S. Lew, Asymptotic expansion of a class of integral transforms with algebraically dominated kernels, J. Math. Anal. Appl. 35 (1971), 405-433.

5. W. Hurewicz, Lectures on ordinary differential equations, Wiley, New York, 1958.

6. D. S. Jones, Generalized functions, McGraw-Hill, London, 1966.

7. E. L. Koh and A. H. Zemanian, The complex Hankel and I-transformations of generalized functions, SIAM J. Appl. Math. 16 (1968), 945-957.

8. G. Köthe, Topological vector spaces, vol. 1, Springer-Verlag, New York, 1969.

9. H. A. Lauwerier, Asymptotic analysis, 2nd ed., Mathematisch Centrum, Amsterdam, 1974.

10. M. J. Lighthill, Fourier analysis and generalized functions, Cambridge Univ. Press, Cambridge, 1958.

11. J. P. McClure and R. Wong, Explicit error terms for asymptotic expansions of Stieltjes transforms, J. Inst. Math. Appl. 22 (1978), 129-145.

12. , Exact remainders for asymptotic expansions of fractional integrals, J. Inst. Math. Appl. 23 (1979), 139-147.

13. F. W. J. Olver, Asymptotics and special functions, Academic Press, New York, 1974.

14. L. Schwartz, Théorie des distributions, Hermann, Paris, 1966.

15. P. N. Shivakumar and R. Wong, Asymptotic expansion of multiple Fourier transforms, SIAM J. Math. Anal. 10 (1979), 1095-1104.

16. R. Wong, Error bounds for asymptotic expansions of Hankel transforms, SIAM J. Math. Anal. 7 (1976), 799-808.

17. __ Explicit error terms for asymptotic expansions of Mellin convolutions, J. Math. Anal. Appl. (to appear).

18. , Distributional derivation of an asymptotic expansion, Proc. Amer. Math. Soc. 80 (1980), $266-270$

19. __ Error bounds for asymptotic expansions of integrals, SIAM Rev. 22 (1980).

20. A. H. Zemanian, The distributional Laplace and Mellin transformations, SIAM J. Appl. Math. 14 (1966), 41-59.

21. __ Generalized integral transformations, Interscience, New York, 1966.

22. D. S. Jones, Generalized transforms and their asymptotic behavior, Philos. Trans. Roy. Soc. London Ser. A 265 (1969), 1-43.

Department of Mathematics, California State Polytechnic University, San luis Obispo, CALIFORNIA 93407 